\title{
Rigidity of reinforced concrete structures in the presence of different cracks
}

\author{
Igor Iakovenko ${ }^{1 *}$, Vladimyr Kolchunov ${ }^{1}$, and Ianina Lymar $^{2}$ \\ ${ }^{1}$ National Aviation University, 03058, Cosmonaut Komarov Avenu, 1, Kyiv, Ukraine \\ ${ }^{2}$ Limited Liability Company "V. Shimanovsky UkrRDISteelconstruction", 02660, Osvoboditelei \\ Avenu, 1, Kyiv, Ukraine
}

\begin{abstract}
It is proposed a method for rigidity calculating of reinforced concrete structures in the presence of cracks, suitable for rod and flatstrained concrete composite structures. It is based on the operating conditions and includes a new, more complete classification of the various cracks, models of a special crack, the calculation of the two-console model; a special cantilever model to determine the parameters of the joint between the concrete; calculation model of the block with the working section at the beginning and end of the crack to determine the horizontal (vertical) projections of various cracks with the involvement of analytical relationships. They are based on the extremum of a function of many variables and Lagrange multipliers, as well as attracting level model of multi-level development of the various cracks, which allow to find the distance between the cracks and width of their disclosure, with considering the effect of discontinuities. This effect can greatly simplify the process of determining the rigidity of reinforced concrete structures (including composite ones), despite the complexity and diversity of the crack pattern.
\end{abstract}

\section{Review of recent studies and publications}

The task of constructing a method for calculating the rigidity of reinforced concrete structures (including composite ones) is already very complicated, because the basic hypothesis of the solid deformable body mechanics (the continuity hypothesis) is not applicable here - the continuity is disturbed by the presence of macrocracks, which greatly reduces their rigidity $[1-3]$.

The analysis shows [1-3], that the solution of the indicated problem is highly disputable and multi-vector. Here, quite complex adjacent tasks of determining the pattern of formation, development and crack opening are included [4]. For example, the values of the crack width opening are measured with a microscope in experiments, and the calculation is carried out with the aid of a rather rough calculating apparatus. It is not necessary to expect the coincidence of experimental and theoretical data in which case. The distances between adjacent cracks are not a functional one, but a discrete parameter, and fracture levels, as shown by experimental studies, can be not one but several $[1,4,5]$. However, despite the polarity of opinions, points of their contact have been found. So, it is generally accepted

*Corresponding author: i2103@ukr.net 
that the calculated dependencies should have an engineering-visual form, a clear physical meaning that allows them to be used more efficiently and creatively in engineering practice, which requires the solution of far from trivial problems of an applied nature.

On the other hand, it is hardly necessary to resort to categorical formulations, for example, with a complete denial of the use of integro-differential equations, which lead, as a rule, to engineering-immense results. This occurs only in the case of formalized approximate solutions of such equations. Here, reaching the level of their analytical solvability is important to be able to obtain very "elegant" solutions.

\section{Basic material and results}

It is proposed a new classification of basic cracks in reinforced concrete structures (including composite ones) on the basis of an in-depth study of the nature and nature of the crack formation $[1,5]$. It is based on the geometric, force (deformation) and interspersed concentration of the stress-strain state, with the corresponding sources-concentrators.

The proposed calculation methodology is based on the following working assumptions:

1. The formation of the subsequent level of cracks occurs after the concrete fibers stretched by the fibers have been reached along the axis of the transverse (longitudinal) reinforcement of limiting deformations $\varepsilon_{b t, u}$. There may be several levels of cracking. The development of various cracks occurs along trajectories approaching straight lines in the reinforced concrete structures (including composite ones).

2. The opening of a crack is considered as the accumulation of relative mutual displacements of reinforcement and concrete in areas located on both sides of the fracture. The effect associated with violation of the continuity of concrete is taken into account [1] in this case.

3. Classification of basic cracks is introduced. In the reinforced concrete structures (including composite ones), the following basic cracks can occur: 1) cracks developing to zones or from zones of geometric concentration of stress-strain state (in places where crosssectional dimensions change, input concentration angles, concentration from non-circular holes, etc.); 2) cracks developing to zones or from zones of concentration of force and deformation loading (the location of support reactions and concentrated forces, the place of change in the intensity of loading along the contour of the structure, the place of deformation loading from subsidence, the type of loading is of particular importance, bending, shear, compression-tension); 3) longitudinal cracks developing in zones of intermedial strain concentration (in the seams between concrete in reinforced concrete composite structures, along longitudinal reinforcement in anchoring zones, etc.). In the building practice (for example, in complex engineering-geological conditions), the most frequent schemes are from force and deformation loading, which, as a rule, cause the imposition of various cracks [6].

4. The actual crack is replaced by the proposed model (Fig. 1) by means of the finite element (FE) casing in the PC "Lira-CAD" [7, 8] and the deformation action $\Delta=a_{\mathrm{crc}, \mathrm{j}}$ along the crack profile (the discontinuity effect [1] is introduced by the actual crack profile, and with the help of the formula for determining the crack opening width).

5. A special design two-element cantilever model is used to compute (Fig. 2). The sequential iterative analysis is performed with the help of the stress-strain state of flat cantilever elements. They are located along the fracture path are realized by means of a "shear" and deformation effect that takes into account the discontinuity effect of concrete $[7,8]$. 


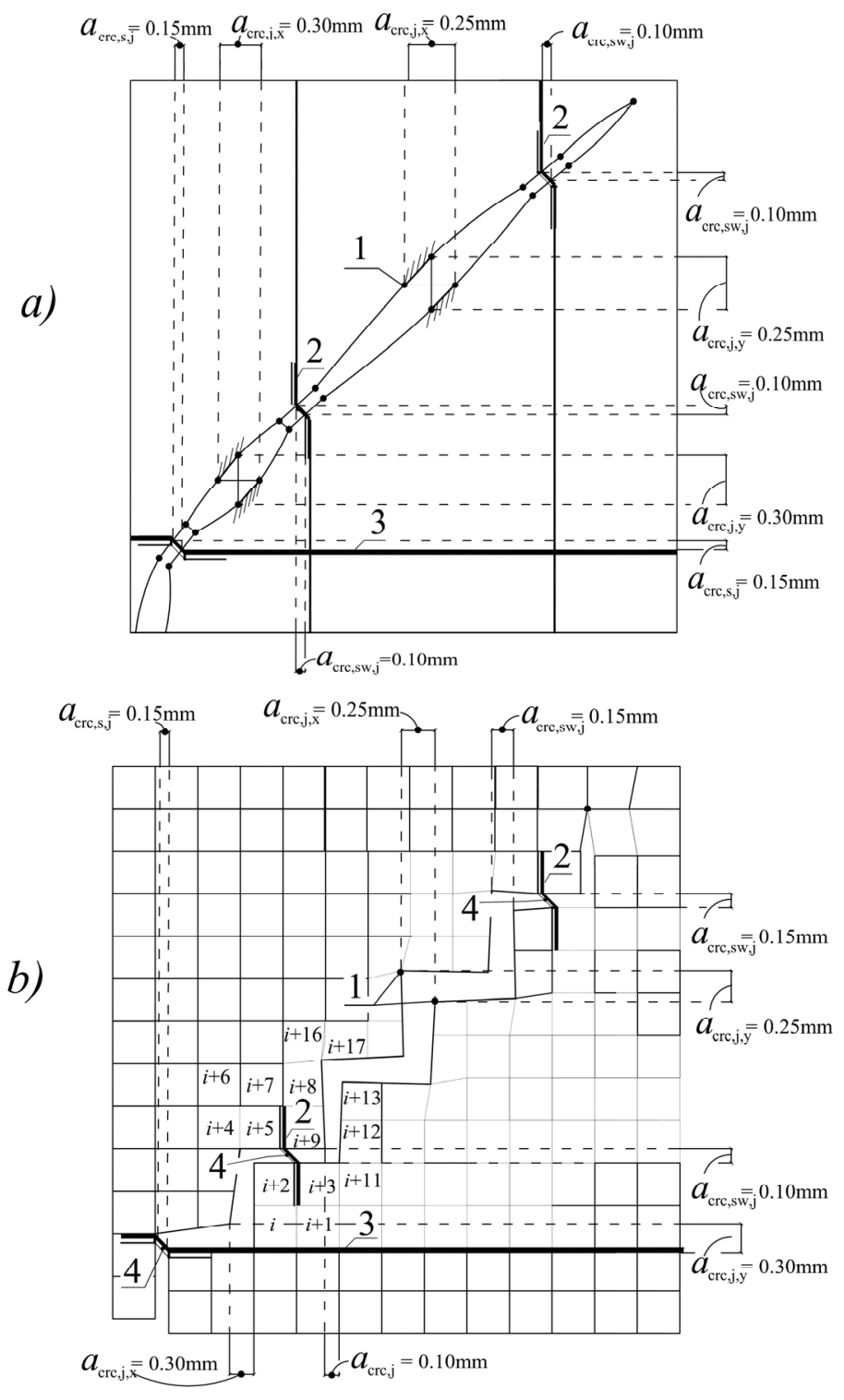

Fig. 1. The proposed model of a crack: $a$ - real crack; $b$ - the crack modeled by means of FE embroidering and deformation effect $\Delta=a_{c r c, j} ; 1$ - crack; 2 - transverse reinforcement and its simulation with $201 \mathrm{FE} ; 3$ - longitudinal reinforcement and its simulation with $201 \mathrm{FE} ; 4$ - possible closure of the fracture and its simulation using $255 \mathrm{FE}$

6. A special design two-element cantilever model is used to calculate the composite structures [9], which makes it possible to determine the parameters of the seam $(G(\lambda), E(\lambda)$, $\mu(\lambda)$ in two rows of small size FE's adjacent to the seam) between the concrete).

It is based on the experiments of shearing the seam of prisms with different concretes, including reinforcing rods in the seam between concrete. At the same time, the capabilities of Lira-SAPR software are used to determine the stress-strain state of the FE (including those adjacent to the seam). 


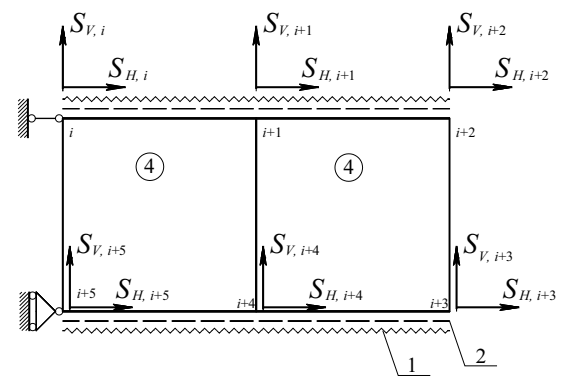

a)

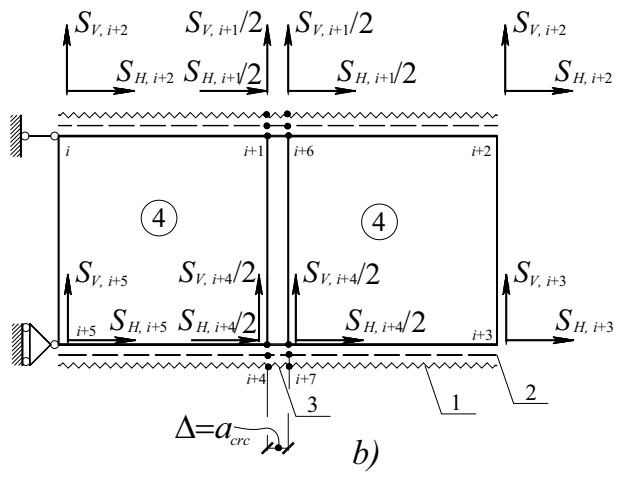

b)

Fig. 2. The construction of a two-element model: $a$-the design scheme of a two-element model without embroidering; $b$ - the design scheme of the two-element model after embroidering; $1-255$ FE before embroidering; 2 - $201 \mathrm{FE} ; 3$ - $255 \mathrm{FE}$ after embroidering; 4 - $233 \mathrm{FE}$

7. The projections of various cracks on the horizontal (vertical) are sought on the basis of the block model with the calculated cross-sections I-I and II-II (Fig. 3), passing through

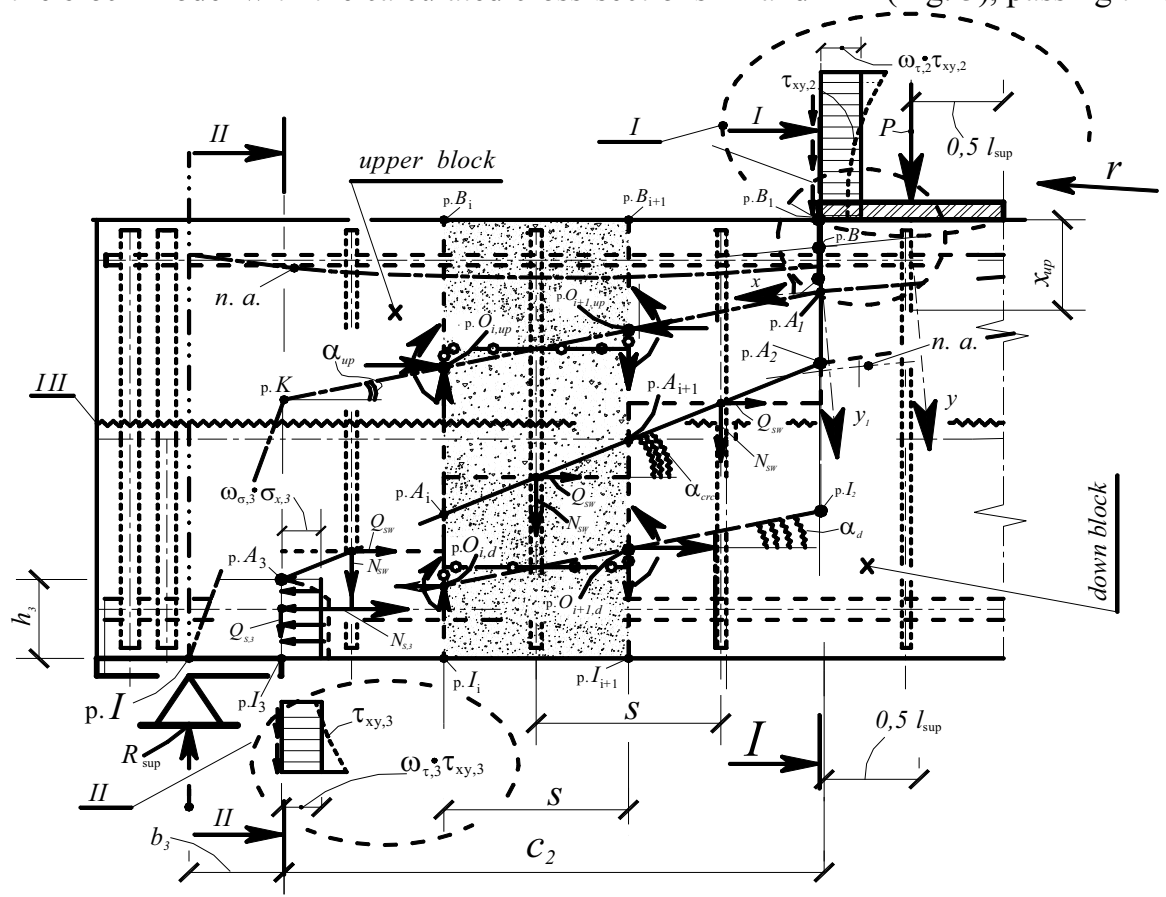

Fig. 3. The calculation scheme for blocks with cross sections I-I and II-II to determine the projection of an inclined crack in a plane-stressed reinforced concrete structure (including composite ones): I and II - diagrams of tangential stresses in sections I-I and II-II, respectively; III - the seam joint between concrete

the beginning and end of the crack (refined in the iteration process). One of these sections, as a rule, is attached to the largest force - the support reaction $R_{\text {sup }}$ or goes to one of the faces of the structure with the use of analytic dependences [4], based on the extremum of the function of several variables and Lagrange multipliers.

8. A multi-level process of development of various cracks takes place in plane-stressed reinforced concrete structures (including composite ones) [4], which is determined by special design models (Fig. 4) of their level appearance. It allows finding the distances 
between cracks and the width of their opening, taking into account the effect of discontinuity.

In this case,

$$
\varepsilon_{\mathrm{bt}}(y)=\varepsilon_{\mathrm{sw}} \cdot E_{\mathrm{sw}} \cdot A_{\mathrm{sw}} \cdot \frac{1}{D_{13}}-\varepsilon_{\mathrm{sw}}(y) \cdot E_{\mathrm{sw}} \cdot A_{\mathrm{sw}} \cdot \frac{1}{D_{13}}+\frac{D_{14}}{D_{13}} \cdot y+\frac{D_{15}}{D_{13}},
$$

a)

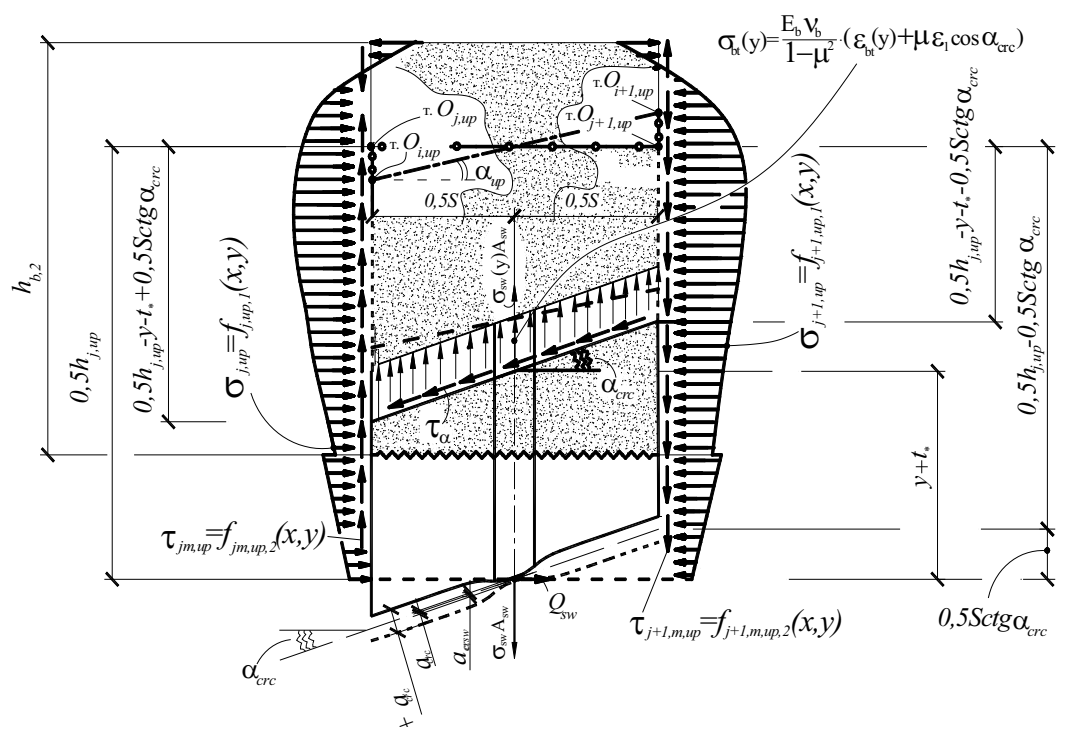

b)

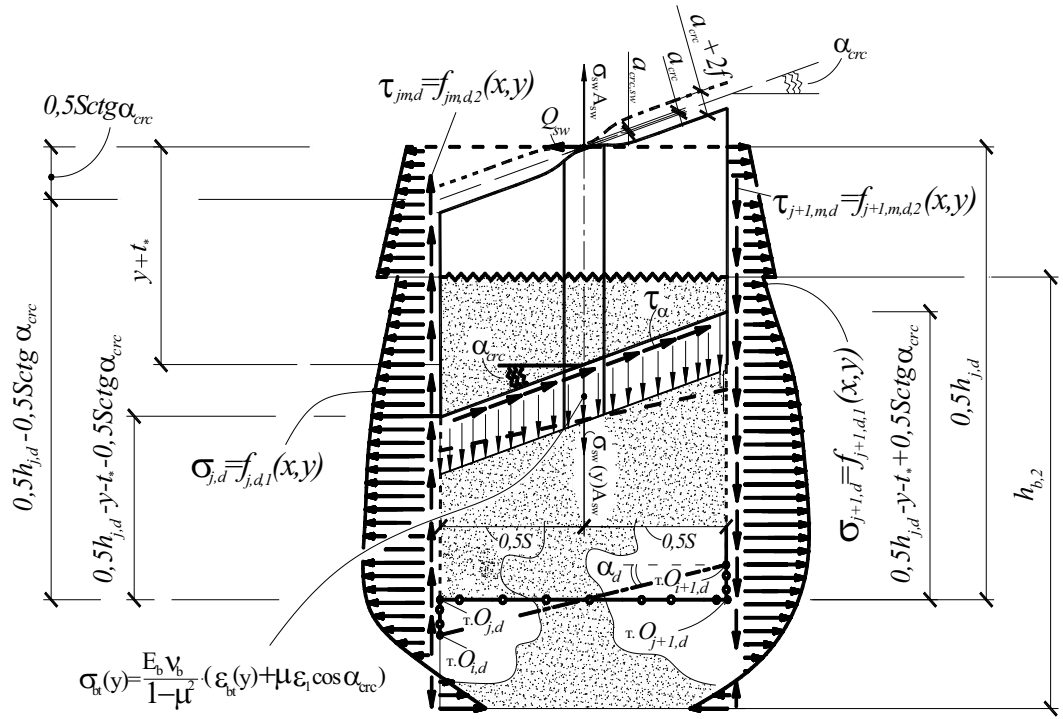

Fig. 4. The level model for determining the deformations of tension concrete $\varepsilon b t(y)$ along the axis of transverse reinforcement of the $i$-th level of formation various cracks and the distance between them in a reinforced concrete composite structure: $a$ - first case; $b$ - second case

Careful analysis $[4,10,11]$ of the pricked cracks and cracks adjacent to the concentrated load, makes possible to reveal a "fan" of inclined cracks adjacent to the concentrated load and to the support (Fig. 5). 
The projection of an inclined crack, whose opening width has the maximum value, is determined from the condition of an extremum of functions $F_{1}=f\left(q_{\mathrm{sw}}, x_{\mathrm{B}}, \sigma_{\mathrm{s}}, x, \sigma_{\mathrm{b}}, \sigma_{\mathrm{sI}}, \sigma_{\mathrm{b}, \mathrm{p}}\right.$ $\left.C_{2}, \lambda_{1}, \lambda_{2}, \lambda_{3}, \lambda_{4}, \lambda_{5}, \lambda_{6}, \lambda_{7}\right)$ of several variables and $F_{2}=f\left(q_{\mathrm{sw}}, x_{\mathrm{B}, 2}, \sigma_{\mathrm{s}, 3}, c_{2}, \lambda_{1}, \lambda_{2}, \lambda_{3}\right)$, respectively, and the resulting conditions for the equality of partial derivatives to zero:

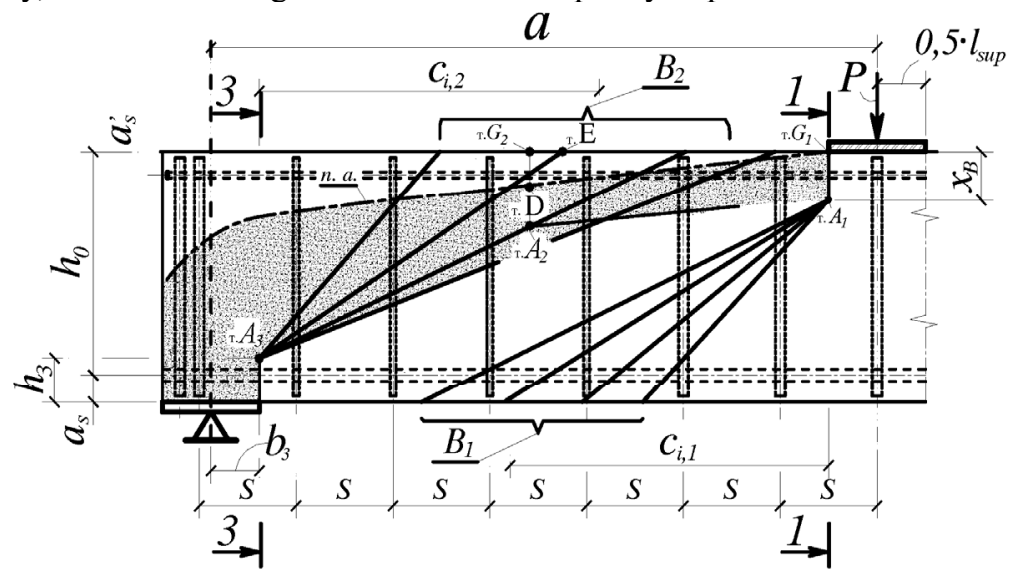

Fig. 5. To analyze the prismatic cracks and cracks adjacent to the concentrated load under flexural loading: $B_{1}$ - "fan" of inclined cracks adjacent to the concentrated load; $B_{2}$ - "fan" of possible inclined cracks adjacent to the support

$$
\begin{aligned}
& \left.\frac{\partial f}{\partial x_{1}}+\lambda_{1} \frac{\partial \varphi_{1}}{\partial x_{1}}+\lambda_{2} \frac{\partial \varphi_{2}}{\partial x_{1}}+\ldots+\lambda_{m} \frac{\partial \varphi_{m}}{\partial x_{1}}=0\right) \\
& \left.\frac{\partial f}{\partial x_{2}}+\lambda_{1} \frac{\partial \varphi_{1}}{\partial x_{2}}+\lambda_{2} \frac{\partial \varphi_{2}}{\partial x_{2}}+\ldots+\lambda_{m} \frac{\partial \varphi_{m}}{\partial x_{2}}=0\right\} \text {. } \\
& \frac{\partial f}{\partial x_{n}}+\lambda_{1} \frac{\partial \varphi_{1}}{\partial x_{n}}+\lambda_{2} \frac{\partial \varphi_{2}}{\partial x_{n}}+\ldots+\lambda_{m} \frac{\partial \varphi_{m}}{\partial x_{n}}=0
\end{aligned}
$$

In this case, having stresses in the longitudinal reinforcement at the cross-section with a dangerous inclined crack (according to the criterion of the maximum opening width at the level of the longitudinal reinforcement axis), it is possible to determine the functional distance between different cracks along the axis of the longitudinal reinforcement, and also by arranging the stresses in the transverse reinforcement at the cross-section with a dangerous inclined crack (according to the criterion of the maximum opening width at the level of the axis of the transverse reinforcement) along the axes of the transverse reinforcement. As a rule, second-level design schemes are used (Fig. 4, 6).

Then the distance between cracks is calculated by the formula below:

$$
l_{\mathrm{crc}}=\frac{2\left(\ln B_{4}-B t_{*}\right)}{-B}
$$

where parametrs

$$
B_{4}=1+\frac{\sigma_{\mathrm{bt}, \mathrm{c}}}{(K-1) B_{3, *} v_{\mathrm{b}} E_{\mathrm{b}}}+\frac{\varepsilon_{\mathrm{bt}, \mathrm{u}}}{B_{3, *}(K-1)}
$$




$$
B_{3}=\varepsilon_{\mathrm{s}}+\frac{\Delta T}{E_{\mathrm{s}} A_{\mathrm{s}}}-\frac{\sigma_{\mathrm{bt}, \mathrm{c}}}{v_{\mathrm{b}} E_{\mathrm{b}}}-B_{2} ; \quad B_{2}=\frac{\delta Q}{t B},
$$

$B$ is the parameter of bond reinforcement with concrete, is taken equal to

$$
\begin{aligned}
& B=\frac{S_{\mathrm{S}} \cdot G}{K \cdot A_{\mathrm{S}} \cdot E_{\mathrm{S}}} ; \\
& \frac{1}{K}=1+2 \mu \alpha,
\end{aligned}
$$

where

$$
t=\frac{A_{\mathrm{S}} E_{\mathrm{b}}}{2 \mu}, \quad \alpha=\frac{E_{\mathrm{S}}}{E_{\mathrm{b}}}, \quad \mu=\frac{A_{\mathrm{S}}}{b \cdot h_{0}} .
$$

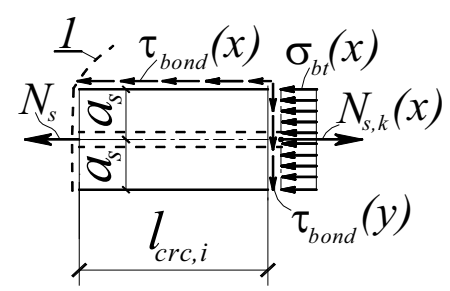

Fig. 6. The calculation scheme for determining the deformations of tensioned concrete $\varepsilon b t(x)$ along the longitudinal reinforcement axis for the $i$-th level of the of inclined cracks formation of the first, second or third types in reinforced concrete structures (including composite)

It should be noted that the field of real numbers by the parameter following restrictions.

$$
0<B_{4}<e^{B \cdot t_{b}},
$$

Comparing the functional and level values of $l_{\mathrm{crc}}$, an analysis is performed of the possible realization of the appearance of subsequent fracture levels.

Analysis of formula (6) shows that an increase in deformations in the reinforcement with increasing load causes a decrease in the distance between the cracks. At the same time, the appearance of a new level of crack formation corresponds to the level of loading at which the following inequality is observed.

$$
l_{\mathrm{crc}, \mathrm{i}} \leq \eta \cdot l_{\mathrm{crc}, \mathrm{i}-1}
$$

where $\eta$ is determined from the following relationships (Fig. 7) to the right of the dangerous inclined crack: 

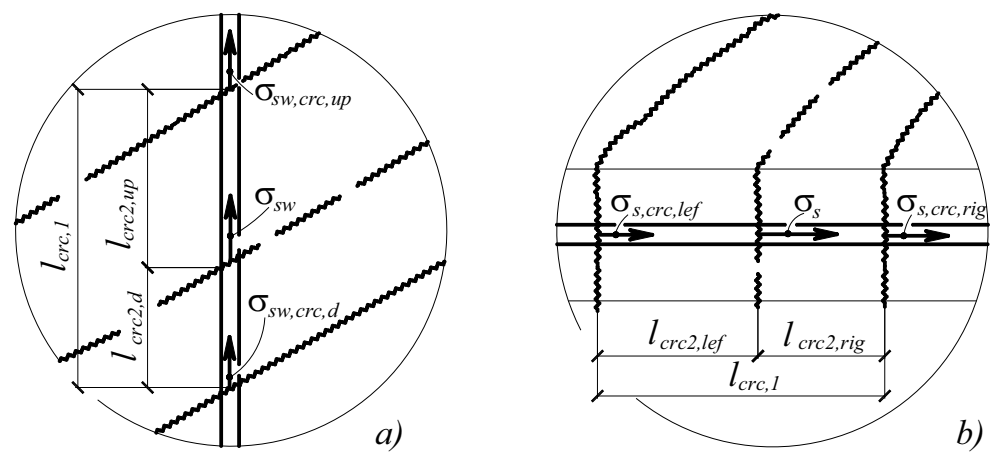

Fig. 7. The location of the adjacent crack of the next level between the two cracks of the previous level: $a$ - along the axis of the transverse reinforcement; $b$ - along the axis of the longitudinal reinforcement

$$
\begin{gathered}
\frac{\sigma_{\mathrm{sw}, \mathrm{crc}, \mathrm{d}}}{\sigma_{\mathrm{sw}, \mathrm{crc}, \mathrm{up}}}=\frac{l_{\mathrm{crc}, 2, \mathrm{up}}}{l_{\mathrm{crc}, 2, \mathrm{~d}}}=\eta ; \\
l_{\mathrm{crc}, 2, \mathrm{up}}+l_{\mathrm{crc}, 2, \mathrm{~d}}=l_{\mathrm{crc}, 1} .
\end{gathered}
$$

Along the longitudinal reinforcement, such relations will take the form (Fig. 7, b):

$$
\begin{aligned}
& \frac{\sigma_{\mathrm{sw}, \mathrm{crc}, \mathrm{rig}}}{\sigma_{\mathrm{sw}, \mathrm{crc}, \text { lef }}}=\frac{l_{\mathrm{crc}, 2, \text { lef }}}{l_{\mathrm{crc}, 2, \mathrm{rig}}}=\eta, \\
& l_{\mathrm{crc}, 2, \text { lef }}+l_{\mathrm{crc}, 2, \text { rig }}=l_{\mathrm{crc}, 1} .
\end{aligned}
$$

Thus, the cracking continues until the moment of destruction is started. In this case, not one is allocated (as is customary in a number of known techniques), but several levels of crack formation:

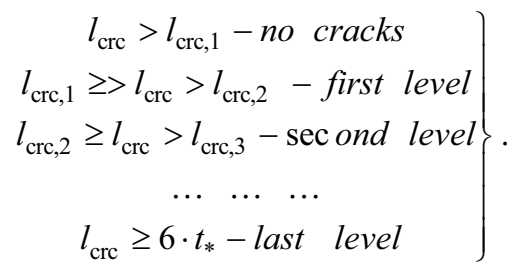

Having a cracking level along the longitudinal and transverse reinforcement of a flatstressed reinforced concrete structures (including composite), a "fan" of various cracks is built up adjacent to the concentrated force and to the support (Fig. 5). The degree of realization of cracks (whether these cracks intersect the lateral reinforcement, or it will be crossed only by a dangerous inclined crack) are determined from consideration of the stress-strain state along the clamps on the basis of the design scheme, the subsequent level shown in Fig. 4, which "closes" to a multi-iterative process, provided using Lira-CAD software.

The distance between the cracks is the most important parameter necessary for determining the crack opening width in reinforced concrete structures. From physical 
considerations, it is logical to use the level (discrete) value in the formula for determining the crack opening width in accordance with inequalities (10) and (14).

Having the stresses in the transverse reinforcement or stresses in the longitudinal reinforcement in the section with the $y$ coordinate, it is possible to find the relative mutual displacements of the reinforcement and concrete:

$$
\varepsilon_{\mathrm{g}}(y)=\varepsilon_{\mathrm{sw}}(y)-\varepsilon_{\mathrm{bt}}(y),
$$

where, using $[1,5]$

$$
\varepsilon_{\mathrm{sw}}(y)=\varepsilon_{\mathrm{sw}}+\frac{\Delta T}{E_{\mathrm{sw}} A_{\mathrm{sw}}}-\frac{S}{A_{\mathrm{sw}} E_{\mathrm{sw}}} \int_{0}^{y} \tau(y) d y .
$$

Here $S_{\mathrm{s}}$ is the perimeter of the reinforcement cross-section; $\boldsymbol{\varepsilon}_{s}$ - deformation of the reinforcement in the crack; and $\Delta T$-resulting conditional tangential stresses at local zone.

The nature of the diagram $\varepsilon_{s w}(y)$, performed with the involvement of the experiments of other authors, shows that, at a certain strain load, the deformations on the areas adjacent to the cracks begin to decrease and even change sign, while deformations in the middle of the section between the cracks continue to increase until at this point new crack. Analysis of the nature of the diagram $\varepsilon_{b t}(y)$ shows the necessity (Fig. 8) of taking into account the deformation effect in crack $[1,4]$.

Substituting $\varepsilon_{b t}(y)$ into the formula (16) and performing the corresponding algebraic transformations, obtain:

$$
\varepsilon_{\mathrm{g}}(y)=\left(\varepsilon_{\mathrm{sw}}+\frac{\Delta T}{E_{\mathrm{sw}} A_{\mathrm{sw}}}-\frac{\sigma_{\mathrm{bt}, \mathrm{c}}}{v_{\mathrm{b}} E_{\mathrm{b}}}-\frac{D_{14}}{D_{13} \cdot B}\right) e^{-\mathrm{B} y}-\frac{D_{14}}{D_{13} \cdot B} .
$$

Here $\frac{\sigma_{\mathrm{bt}, \mathrm{c}}}{v_{\mathrm{b}} E_{\mathrm{b}}}$ corresponds to the deformation of concrete $\varepsilon b t(y)$ in a section located at a distance from $t *$ from the section with a crack (Fig. 8). In this case, the values $\sigma_{b t, c}$ are taken with the minus sign here and in all the below formulas.

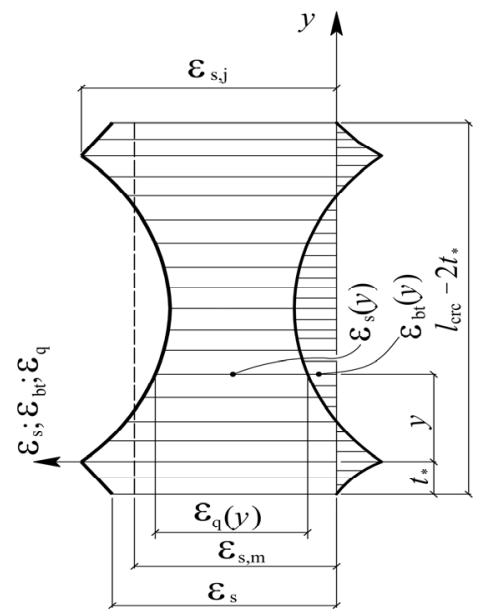

Fig. 8. Diagrams of deformations of concrete $\varepsilon_{b t}(y)$, reinforcement $\varepsilon_{s w}(y)$ and their relative mutual displacements $\varepsilon_{g}(y)$ in the area between inclined cracks in reinforced concrete structures 
Thus, the problem by definition $a_{c r c}$ is reduced to finding the relative mutual displacements $\varepsilon_{g}(y)$ of reinforcement and concrete in different sections between the cracks:

$$
a_{\mathrm{crc}}=2 \int_{0}^{t_{*}} \varepsilon_{\mathrm{g}}\left(y_{1}\right) d y_{1}+\int_{0}^{\eta \cdot l_{\mathrm{crc}}} \varepsilon_{\mathrm{g}}(y) d y+\int_{\eta \cdot l_{\mathrm{crc}}}^{l_{\mathrm{crc}}} \varepsilon_{\mathrm{g}}(y) d y .
$$

After integration and some simplifications, we get:

$$
a_{\mathrm{crc}}=-\frac{2 \Delta T}{G}-\frac{2 B_{a, 2}}{B}-\frac{2 B_{2}}{B} \ln \left(1+\frac{B_{a, 2} \cdot A_{\mathrm{sw}} E_{\mathrm{sw}}}{q_{\mathrm{sw}} S+B_{a, 1} A_{\mathrm{sw}} E_{\mathrm{sw}}}\right) .
$$

The distance between the cracks (3), (10), (14), (15) is the most important parameter necessary for determining the resistance of tensioned concrete between cracks.

Integration $\varepsilon_{s}$ within the distance between cracks, makes it possible to obtain the value of the coefficient $\Psi_{s}$ of stretched concrete work account of according to the formula [12]:

$$
\begin{gathered}
\psi_{\mathrm{s}}=\frac{2 \cdot K \cdot B_{3}}{\varepsilon_{\mathrm{s}} \cdot l_{\mathrm{crc}} \cdot B} \cdot\left[1-e^{-B \cdot\left(0.5 l_{\mathrm{crc}}-t_{*}\right)}\right]+\frac{2}{\varepsilon_{\mathrm{s}} \cdot l_{\mathrm{crc}}}\left(\varepsilon_{\mathrm{s}}+\frac{\Delta T}{E_{\mathrm{s}} A_{\mathrm{s}}}-K \cdot B_{3}\right) \cdot\left(0,5 l_{\mathrm{crc}}-t_{*}\right)+ \\
+\frac{\delta Q \cdot K}{\varepsilon_{\mathrm{s}} \cdot l_{\mathrm{crc}} \cdot B \cdot t_{*}}\left(0,5 l_{\mathrm{crc}}-t_{*}\right)^{2}+\frac{2 \cdot \varepsilon_{\mathrm{s}} \cdot E_{\mathrm{s}} \cdot A_{\mathrm{s}}+\Delta T}{\varepsilon_{\mathrm{s}} \cdot l_{\mathrm{crc}} \cdot E_{\mathrm{s}} \cdot A_{\mathrm{s}}} \cdot t_{*} .
\end{gathered}
$$

Obtained parameters according to (3), (10), (14), (15), (20), (21) account the effect of a number of important factors, such as deformation of reinforcement in the cross section with a crack, parameter of bond reinforcement with the concrete, geometrical sectional characteristics of concrete and reinforcement, deplanation on the surface of concrete structures with respect to the radius of the boundary layer, the deformation effect (appearing in reinforced concrete member after discontinuity) centered relative conditional mutual displacements concrete and reinforcement and, where necessary, the effect of transverse force.

\section{Conclusions}

On the basis of the proposed design model for the width of the opening of inclined cracks in reinforced concrete structures (including composite ones), it was also possible to refine the parameters of the stress-strain state of reinforced concrete structures in the presence of various cracks, taking into account the discontinuity effect [1]. This served as the basis for the development of a method for calculating the stiffness in reinforced concrete structures (including composite ones), which is reduced to the following algorithm, considered in two versions with the use of the PC Lira-CAD software complex.

The first variant is performed without changing the given order and the flat FE numbers. In FE, adjacent to implicit cracks, their thickness decreases. The operation of each pair of CE's is calculated twice using a two-element cantilever model (TECM) (Fig. 2): before embroidering the FE $\left(W_{l}\right)$ and after embroidering the FE from the crack opening taking into account the effect of discontinuity $\left(W_{2}\right)$ (the field reinforcement is replaced by two fibreboard FE). Efforts in the TECM nodes are determined from the nonlinear calculation of the entire plane-stressed structure. The displacement of the nodes is determined from the calculation of the TECM with the forces exerted in the nodes (Fig. 2). As a result, the new thickness of FE's adjacent to the crack is determined by the formula: 


$$
b_{i}=\frac{W_{1}}{W_{\mathrm{i}}} \cdot b_{1} .
$$

The calculation algorithm assumes the presence of an iterative process, which is regulated by the accuracy achieved in the thickness of the specified FE.

In the second variant, the rigidity of reinforced concrete structures is determined using by special method of modeling the sheer cracks - gaps, when they are opened and closed Fig. 1, $b$, taking into account the effect of discontinuity and the incompatibility of deformations of reinforcement and concrete. Reinforcing rods are modeled by an additional $201 \mathrm{FE}$, and a possible closure of a crack, - concrete $255 \mathrm{FE}$.

Thus, a method is proposed for calculating the rigidity of reinforced concrete structures in the presence of cracks, suitable for rod and flat-concrete reinforced concrete structures (including composite ones), based on working assumptions on a new, most complete classification of various cracks; special model of cracks; two-element cantilever (console) model; the special console model for determining the parameters of the seam between concrete (in the case of a composite structure); block calculation model with working sections, passing through the beginning and end of the crack to determine the projections of various cracks to the horizontal (vertical) with the use of analytical dependencies based on the extremum of the function of many variables and Lagrange multipliers, and also involving level models of the multilevel development of various cracks that allow to find the distances between the cracks and the width of their opening, with the discontinuity effect. It allows to substantially simplify the process of determining the hard of reinforced concrete structures, despite the complexity and diversity of the pattern of cracking.

\section{References}

1. A.B. Golyshev, V.I. Kolchunov. The resistance of reinforced concrete, 432 p. (Osnova, Kyiv, 2009)

2. Li Yongzhen. Final Report Predicting of the Stiffness of Cracked Reinforced Concrete Structures, 147 p. (2010)

3. P. N. Saavedra, L. A. Cuitino. Crack detection and vibration behaviour of cracked beam, Computers and Structures, 79, pp. 1451 - 1459 (2001)

4. H.Z. Bashirov, V.I. Kolchunov, V.S. Fedorov, I.A. Iakovenko. Reinforced concrete structures of buildings and structures, 248 p. (ASV, Moskow, 2017)

5. T.V. Tugay. The main results of experimental studies of flat-strained stiffness of reinforced concrete composite structures, Resource-economical materials, structures, buildings, 29, pp. 369-375 (Rivne, 2014).

6. A.B. Golyshev, V.I. Kolchunov, I.A. Iakovenko. Resistance of reinforced concrete structures, buildings and structures erected in complex engineering-geological conditions, 371 p. (Talcom, Kyiv, 2015)

7. V.I. Kolchunov, N.G. Maryenkov, T.V. Tugai, I.A. Iakovenko. Resistance of planestressed walls to a skew in the presence of a diagonal crack with the use of the Lira-Pro software complex. Academic journal. Industrial Machine Building, Civil Engineering Poltava National Technical Yuri Kondratyuk University, 1 (43) (Poltava, 2015)

8. V.I. Kolchunov, T.V. Tugai, I.A. Iakovenko, N.G. Maryenkov. Calculation of the stiffness of plane-stressed walls with the use of PC "Lira-Pro" by the method of new norms. City and territorial planning, 53, pp. 209-221.

9. V.I. Kolchunov, N.G. Maryenkov, T.V. Tugai, I.A. Iakovenko. Method for determination of stiffness of flat-stressed and rod-shaped reinforced concrete composite structures under seismic influences // Industrial and civil construction, №2, pp. 12-15 (Moscow, 2014) 
10. P. Rosko and A. Bekw. Reinforced concrete shear wall: structural element - finite element. Vienna University of Technology. Center of Mechanics and Structural, Dynamics Institute for Building Construction and Technology, 7p (Vienna, 2014).

11. Yo. Yamamoto, H. Nakamura, I. Kuroda and N. Furuya. Simulation of crack propagation in rc shear wall using a $3 \mathrm{~d}$ rigid-body-spring model with random geometry. VIII International Conference on Fracture Mechanics of Concrete and Concrete Structures, 12 p. (Toledo - Spain, 2013)

12. I.A. Iakovenko, G.K. Bidzhosyan. New dependencies for estimating the resistance of stretched concrete between cracks in compound eccentrically compressed reinforced concrete structures. Bulleten of Pridniprovsk State Academy of Construction and Architecture, 9, pp. 9-15 (Dnipropetrovsk, 2012). 